\title{
20 años de entrenamiento en espirometría en América Latina
}

\author{
Rogelio Pérez-Padilla,凶 Luis Torre-Bouscoulet, Laura Gochicoa-Rangel, Juan Carlos Vázquez-García
}

Instituto Nacional de Enfermedades Respiratorias Ismael Cosío Villegas, Ciudad de México.

Trabajo recibido: 14-XII-2016; aceptado: 16-XII-2016

\section{INTRODUCCIÓN}

En 2015 se cumplieron 20 años de la realización de cursos estandarizados de espirometría en Latinoamérica. Los cursos han estado respaldados desde el año 2004 por la Asociación Latinoamericana del Tórax (ALAT), siendo el primer curso formal durante el IV Congreso de ALAT en Buenos Aires, Argentina, del 9-10 de octubre del 2004. Los coorganizadores fueron el Dr. Rogelio Pérez Padilla (RPP) y el Dr. Robert Crapo (figura 1). ${ }^{1} \mathrm{La}$ intención de aquel primer curso fue entrenar a futuros profesores de diversas regiones del continente para que cada uno pudiera reproducir los cursos en sus propios países manteniendo la misma metodología. Brasil ha tenido cursos de entrenamiento independientes respal-

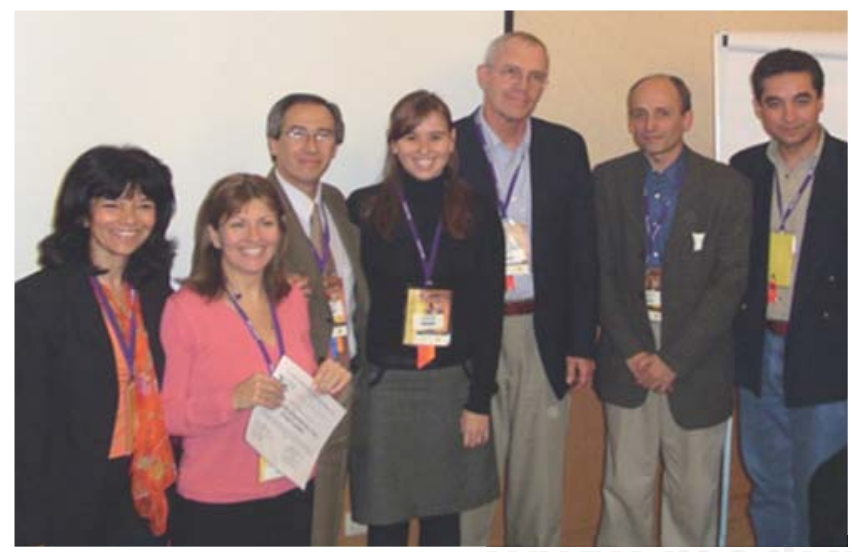

Figura 1. Algunos de los participantes del primer curso de espirometría para entrenadores de la Asociación Latinoamericana del Tórax en Buenos Aires el 9-10 de octubre del 2004. Están de izquierda a derecha: María Nelly Márquez (instructora), Adriana Muiño (instructora), Carlos Torres, Fernanda Rosa, Robert Crapo (codirector fallecido), Rogelio PérezPadilla (codirector) y el Dr. Juan Carlos Vázquez (instructor). dados por la Sociedad Brasileña de Neumología (SBP) a los cuales no nos referiremos.

\section{ANTECEDENTES}

El 17 y 18 de junio de 1995 se realizó el primer curso estandarizado de espirometría en el Instituto Nacional de Enfermedades Respiratorias (INER) en la Ciudad de México. Aquel curso se llevó a cabo siguiendo los procedimientos del Instituto Nacional para la Seguridad y la Salud Ocupacional de los Estados Unidos de Norteamérica (National Institute for Occupational Safety and Health, NIOSH). Gracias a una colaboración vigente con el Dr. Jonathan Samet y el Dr. David Coultas -el último, director del curso $\mathrm{NIOSH}$ - se certificaron en marzo de 1995 los primeros espirometristas mexicanos (RPP, Raúl Sansores y Teresa Fortoul) en Albuquerque, Nuevo México. En los primeros cursos se utilizó una traducción al castellano del manual vigente de NIOSH que incorporaba mediciones manuales para algunas de las variables espirométricas.

El curso se siguió impartiendo regularmente con lo que se desarrollaron presentaciones estandarizadas y material original en forma de manuales. En 1996 se aceptó formalmente por NIOSH la sede del INER para los cursos de espirometría (sede número 107) con RPP como director. El curso 107 del INER sigue vigente hasta la actualidad. ${ }^{2}$ Desde 1998, se imparte regularmente como una actividad regular de educación continua. Desde su fundación, en el INER se ha impartido un total de 73 cursos de certificación con más de 1,200 espirometristas certificados. Actualmente, existen dos sedes en la Ciudad de México (107 y 122), y fue por muchos años la única sede aprobada fuera de los Estados Unidos, ya ahora con varios profesores titulares evaluados y aceptados por NIOSH (RPP, 
JCV, LTB, LGR) que imparten en México al menos 5-6 cursos al año. El grupo de trabajo del INER ha estado involucrado con la traducción al castellano de los manuales de espirometría de NIOSH. Sin embargo, con el tiempo se han desarrollado materiales propios de gran aceptación que prueban la gran experiencia científica y docente que ha ganado el grupo. Como parte de su entrenamiento, todos los residentes de neumología y neumología pediátrica y los alumnos de la Escuela de Terapia Respiratoria de nuestro centro, deben aprobar el curso.

\section{PLATINO Y LOS CURSOS DE ESPIROMETRÍA}

Con la planeación del proyecto PLATINO (Proyecto Latinoamericano sobre Investigación en Obstrucción Pulmonar) para definir la prevalencia de enfermedad pulmonar obstructiva crónica en cinco ciudades latinoamericanas, se realizó un entrenamiento estandarizado al personal espirometrista por el mismo equipo de trabajo (RPP y JCV) entre 2002 (Saõ Paulo), 2003 (Montevideo, Santiago y México) y 2004 (Caracas). En este proyecto participaron 64 técnicos de espirometría, de los cuales 47 no tenían experiencia previa en espirometría. La gran mayoría de las más de 5 mil pruebas que se realizaron alcanzaron o superaron el estándar internacional de calidad. Además, este programa permitió depurar el material educativo y el proyecto en sí generó información abundante sobre control de calidad espirométrica ${ }^{3-7}$ así como manuales de entrenamiento que fueron base de las presentaciones oficiales del curso y de los manuales de entrenamiento que se han utilizado, todos incluyendo datos originales. ${ }^{8}$ En el año 2004 se propuso el Curso de Espirometría como un proyecto mayor de educación de la ALAT. En el seno del IV congreso ALAT en Buenos Aires, Argentina, se organizó el Primer Curso de Entrenadores de Espirometría con RPP y Robert Crapo como Profesores Titulares del curso (figura 1).

\section{DESARROLLO DE LOS CURSOS}

El curso de entrenamiento para espirometristas está centrado, como el original de $\mathrm{NIOSH}$, en los aspectos técnicos de la prueba y tiene un componente de conferencias, pero la parte central es la práctica en la cual los participantes se realizan pruebas espirométricas unos a otros buscando la calidad óptima, después de calibrar o verificar la calibración de los equipos de espirometría. Finalmente hay un examen escrito y uno práctico que consiste en la realización de una prueba espirométrica de buena calidad.
Se ha realizado el curso exitosamente, auspiciado por ALAT y los organizadores locales en el congreso anual de la Federación Centroamericana y del Caribe que agrupa a Centroamérica, Cuba, República Dominicana y más recién Venezuela. También en varias reuniones Latinoamericanas de ALAT, y en congresos nacionales de muchos países latinoamericanos. En total se han realizado hasta el momento aproximadamente 40 cursos en América Latina con 30 estudiantes en cada uno en promedio.

\section{DESARROLLO DE CURSOS LOCALES}

Se han realizado cursos locales en Argentina (Dr. Orlando López Jove y Dr. Eduardo de Vito), que se realiza con regularidad, en Uruguay (Dra. Adriana Muiño y Dra. María Nelly Márquez) y otros ofrecidos inconstantemente ${ }^{9}$ similares al original de ALAT y se podrían realizar en la mayoría de los países ya que cuentan con un número suficiente de personal entrenado. En Centroamérica se ha iniciado el curso itinerante de espirometría en el cual se maneja la misma metodología NIOSH.

Uno de los aspectos importantes es que el curso ha sido tomado por individuos con una gran variedad de entrenamientos: enfermeras, técnicos en inhaloterapia, terapia física o rehabilitación, neumólogos, pediatras, pediatras neumólogos, y un gran número de médicos con especialidad en medicina ocupacional, demostrando que las habilidades básicas para realizar espirometrías no requieren una preparación sofisticada y menos un licenciatura universitaria.

\section{COMENTARIO Y PERSPECTIVAS}

Los cursos de espirometría, con una considerable estandarización y experiencia en trabajo de campo a partir del estudio PLATINO han sido extraordinariamente exitosos. Al final del curso, la inmensa mayoría de los asistentes obtienen la calificación más alta en cuanto a su organización, interés y aprendizaje, facilitado por un ambiente amable y relajado que requiere interacción y colaboración entre los participantes.

En buena medida se ha aprovechado la larga experiencia de los cursos de NIOSH enriquecida por la experiencia latinoamericana. Han Ilenado un hueco de capacitación que se requería urgentemente y que aprecian enormemente en los diferentes sitios donde se ha impartido. Una característica que sin duda ha contribuido al éxito de los cursos es que en muchas ocasiones éstos se desarrollan durante las actividades académicas de algún congreso o reunión local que hace que los asistentes, con el mismo gasto derivado de su traslado al congreso, se benefician de ambas activida- 
des. La experiencia de muchos años nos dice que el modelo de dos días completos es exitoso y suficiente para realizar pruebas de buena calidad técnica, lo que puede ser aplicado de inmediato en sus centros de trabajo, aunque es un tiempo insuficiente para agotar todos los pormenores de la espirometría.

Un aspecto clave es que el material educativo se les entrega a los participantes con dos semanas de anticipación, lo que permite que los alumnos consoliden conceptos durante las conferencias y desarrollan la habilidad y destreza para realizar la espirometría.

Debido a demandas de alumnos se desarrolló posterior un curso estandarizado de interpretación espirométrica dedicado a médicos generales que también ha sido muy exitoso y, últimamente, un curso sobre pruebas de función pulmonar que incorpora DLCO, medición de volúmenes pulmonares y caminata de seis minutos siguiendo un esquema similar, que incorpora no sólo conferencias sino que implica también talleres con demostraciones técnicas y la participación activa de los alumnos.

Este entrenamiento llena un hueco clínico considerable ya que la espirometría es una prueba importante para valorar la función mecánica respiratoria en cualquier enfermedad ${ }^{10}$ con una subutilización mundial, pero es un criterio diagnóstico de la enfermedad pulmonar obstructiva crónica (EPOC) ${ }^{11}$ en donde se ha demostrado que por carecer de espirometría aproximadamente el $90 \%$ de los pacientes no se diagnostican, y casi la mitad de los pacientes con diagnóstico médico de EPOC no cumplen el criterio de obstrucción. ${ }^{12}$ También la espirometría es criterio de valoración de gravedad de una crisis asmática y del seguimiento de los pacientes ${ }^{13}$ que aporta criterios objetivos a la presencia de síntomas y de signos clínicos.

\section{REFERENCIAS}

1. Vázquez-García JC. Cursos de espirometría ALAT. 2013. Fecha de consulta: 14-VI-2016. Accesible en: http://www. congresosalat.org/images/stories/3_pdf/cursos_ALAT/ espirometria/programa_cursosespirometriaALAT.pdf

2. NIOSH. Existing spirometry NIOSH-aproved courses. Access date: 2016 June 15. Available from: http://www. cdc.gov/niosh/topics/spirometry/approved-course.html

3. Pérez-Padilla R, Valdivia G, Muiño A, et al. Spirometric reference values in 5 large Latin American cities for subjects aged 40 years or over. Arch Bronconeumol 2006;42(7):317-325.

4. Pérez-Padilla R, Vázquez-García JC, Márquez MN, et al; Latin American COPD Prevalence Study (PLATINO) Team. The long-term stability of portable spirometers used in a multinational study of the prevalence of chronic obstructive pulmonary disease. Respir Care 2006;51(10):1167-1171.

5. Pérez-Padilla R, Bouscoulet LT, Vázquez-García JC, et al; PLATINO Group. Spirometry reference values after inhalation of 200 microg of salbutamol. Arch Bronconeumol 2007;43(10):530-534.

6. Pérez-Padilla R, Hallal PC, Vázquez-García JC, et al; PLATINO Group. Impact of bronchodilator use on the prevalence of COPD in population-based samples. COPD 2007;4(2):113-120.

7. Pérez-Padilla R, Vázquez-García JC, Márquez MN, Menezes AM; PLATINO Group. Spirometry qualitycontrol strategies in a multinational study of the prevalence of chronic obstructive pulmonary disease. Respir Care 2008;53(8):1019-1026.

8. Vázquez-García JC, Pérez-Padilla J. Manual para el uso y la interpretación de la espirometría por el médico. Asociación Latinoamericana del Tórax; 2007. pp.76.

9. ALAT. Cursos fisiopatología ALAT. Access date: 2016 June 16. Available from: https://http://www.alatorax. org/fisiopatologia/cursos-fisiopatologia-alat

10. Vargas-Domínguez C, Gochicoa-Rangel L, VelázquezUncal M, et al. Pruebas de función respiratoria, ¿cuál y a quién? Neumol Cir Torax 2011;70(2):101-117.

11. Vestbo J, Hurd SS, Agustí AG, et al. Global strategy for the diagnosis, management, and prevention of chronic obstructive pulmonary disease: GOLD executive summary. Am J Respir Crit Care Med 2013;187(4):347365. doi: 10.1164/rccm.201204-0596PP.

12. Tálamo C, de Oca MM, Halbert R, et al; PLATINO Team. Diagnostic labeling of COPD in five Latin American cities. Chest 2007;131(1):60-67.

13. Reddel HK, Bateman ED, Becker A, et al. A summary of the new GINA strategy: a roadmap to asthma control. Eur Respir J 2015;46(3):622-639. doi: 10.1183/13993003.008532015.

\section{$\triangle$ Correspondencia:}

Dr. Rogelio Pérez Padilla

Instituto Nacional de Enfermedades Respiratorias Ismael Cosío Villegas.

Calzada de Tlalpan Núm. 4502, colonia Sección XVI, 14080, Del. Tlalpan, Ciudad de México.

Correo electrónico: perezpad@gmail.com

Los autores declaran no tener conflicto de intereses. 